\title{
INDUCED REPRESENTATIONS OF LIE ALGEBRAS. II
}

\author{
NOLAN R. WALLACH ${ }^{1}$
}

1. Introduction. Let ( $B$ be a Lie algebra over a field $K$. A decomposition of $\mathbb{B}$ is a triple $\left(\mathfrak{n}_{1}, \mathfrak{h}, \mathfrak{n}_{2}\right)$ of subalgebras of $\&$ such that $\mathfrak{G}=\mathfrak{n}_{1} \oplus \mathfrak{h} \oplus \mathfrak{n}_{2}$ a vector space direct sum and such that $\left[\mathfrak{h}, \mathfrak{n}_{i}\right] \subset \mathfrak{n}_{i}$ for $i=1,2$. In [5] we showed how one could "induce" (3)-modules from $\mathfrak{h}$-modules in a natural manner. In this paper we prove a useable necessary and sufficient condition that a $\$$-module must satisfy in order that it be "induced" from an $\mathfrak{h}$-module. We apply this method of induction to obtain all well-known simple modules (not necessarily finite dimensional) for semisimple Lie algebras over algebraically closed fields of characteristic 0 .

2. Preliminary results. Let $\&$ be a Lie algebra over a field $K$ with decomposition $\left(\mathfrak{n}_{1}, \mathfrak{h}, \mathfrak{n}_{2}\right)$. Set $\mathfrak{t}=\mathfrak{n}_{1}+\mathfrak{h}$. Let $U(\mathfrak{S})$ be the universal enveloping algebra of $\mathfrak{B}$ and let $U(\mathfrak{f}), U\left(\mathfrak{n}_{1}\right), U(\mathfrak{h})$ and $U\left(\mathfrak{n}_{2}\right)$ be the universal enveloping algebras of $\mathfrak{t}, \mathfrak{n}_{\mathfrak{1}}, \mathfrak{h}, \mathfrak{n}_{2}$ canonically embedded in $U(\mathfrak{S})$. We look upon $U(\mathfrak{S})$ as a left $U(\mathfrak{t})$-module and a right $U(\mathfrak{S})$ module under, respectively, left and right multiplication. Let $W$ be an $\mathfrak{h}$-module. We denote by $\hat{W}$ the $\mathrm{t}$-module with space $W$ and with $\mathfrak{n}_{1} \cdot W=0$. We set $T(W)=\operatorname{Hom}_{U(\mathfrak{l})}(U(\$), \hat{W})$ with the natural left (3)-module structure

$$
(x \cdot f)(g)=f(g x) \quad \text { for } x \in \mathcal{B}, \quad f \in T(W), \quad g \in U(\xi) .
$$

By the Poincaré-Birkhoff-Witt (abbreviated P-B-W) theorem $U(\mathbb{B})$ $=U(\mathfrak{t}) \mathfrak{n}_{2} \cdot U\left(\mathfrak{n}_{2}\right) \oplus U(\mathfrak{l})$ a right $\mathfrak{h}$-module, left $\mathfrak{t}$-module direct sum. Let $\gamma: U(\mathfrak{B}) \rightarrow U(\mathfrak{f})$ be the corresponding $\mathfrak{h}$-module projection. Let $w: W \rightarrow T(W)$ be defined by $w(v)(g)=\gamma(g) \cdot v$ for $g \in U(\xi), v \in W$. It is easy to see that $w(W)=T(W) \circ \gamma$ (see [5]) and is thus isomorphic with $W$ as an $\mathfrak{h}$-module. We set $W^{*}=U(\mathfrak{B}) \cdot w(W) . W^{*}$ is a $(3-$ submodule of $T(W)$ and $W^{*}$ is the "induced" module of $\S 1$. Before proceeding, we introduce one more bit of notation. If $V$ is a left $B$ module and if $M$ is a subalgebra of $(B)$ then set $V^{M}=\{v \in V \mid M \cdot v=0\}$.

Lemma 2.1. Let $W$ be an h-module.

(1) $\left(W^{*}\right)^{n_{2}} \neq(0)$ if $W^{*} \neq(0)$.

(2) $U($ (S) $) \cdot\left(W^{*}\right)^{\mathfrak{n}_{2}}=W^{*}$.

Received by the editors February 1, 1968.

1 This work has been partially supported by National Science Foundation Grant GP-7499. 
(3) $\left(W^{*}\right)^{\mathfrak{n}_{2}} \cap \mathfrak{n}_{1} \cdot W^{*}=(0)$.

(4) $\mathfrak{n}_{1} \cdot W^{*}$ contains no nonzero $\$$-modules.

Proof. $w(\hat{W})=\left(W^{*}\right)^{\mathfrak{n}_{2}}$ and thus (1) is true. Statement (2) is just the definition of $W^{*}$. To prove statement (3) we notice that if $f \in\left(W^{*}\right)^{\mathfrak{n}_{2}}$ then $f \neq 0$ if and only if $f(1) \neq 0$. If $n_{1} \cdot f \in\left(W^{*}\right)^{\mathfrak{n}_{2}}$ for some $f \in W^{*}$ and $n_{1} \in \mathfrak{n}_{1}$ then $\left(n_{1} \cdot f\right)(1)=f\left(n_{1}\right)=n_{1} \cdot f(1)=0$. Thus $n_{1} \cdot f=0$. We have thus proven (3). If $f \in \mathfrak{n}_{1} \cdot W^{*}$ and if $U(\mathcal{B}) \cdot f \subset \mathfrak{n}_{1} \cdot W^{*}$ then $(g \cdot f) \circ \gamma=0$ for all $g \in U(\$)$. In fact, if $(g \cdot f) \circ \gamma \neq 0$ for some $g \in U(\$)$ then $g \cdot f \circ \gamma \in\left(W^{*}\right)^{n_{2}}$ (by the remarks preceding the lemma) and $g \cdot f \in \mathfrak{n}_{1} \cdot W^{*}$ implies that $g \cdot(f \circ \gamma) \in \mathfrak{n}_{1} \cdot W^{*} \cap\left(W^{*}\right)^{\mathfrak{n}_{2}}=(0)$, which is a contradiction. We thus have $0=(g \cdot f)(\gamma(1))=(g \cdot f)(1)=f(g)$ for all

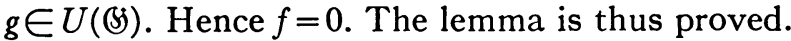

Lemma 1 implies that as an h-module, $W^{*}=\left(W^{*}\right)^{\mathfrak{n}_{2}} \oplus \mathfrak{n}_{1} \cdot W^{*}$. Let $P: W^{*} \rightarrow\left(W^{*}\right)^{\mathfrak{n}_{2}}$ and $Q: W^{*} \rightarrow \mathfrak{n}_{1} \cdot W^{*}$ be the corresponding $\mathfrak{h}$-module projections.

Lemma 2.2. Let $V$ be a (S-module and let $W$ be an $\mathfrak{h}$-module. Then the map $\operatorname{Hom}_{U(\mathbb{G})}\left(V, W^{*}\right) \rightarrow \operatorname{Hom}_{U(\mathfrak{b})}(V, W)$ given by $f \rightarrow P$ of is 1-1. (Here $W^{* \mathfrak{n}_{2}}$ is identified with $W$ as an $\mathfrak{h}$-module.)

Proof. If $P \circ f=0$ then $f(V) \subset \mathfrak{n}_{1} \cdot W^{*}$. Lemma 1 (4) tell us that $f(V)=(0)$. Thus $f=0$.

We note that Lemma 2 is formally half of the Frobenius reciprocity theorem. We conclude this section with a useful sufficient condition for simplicity of $W^{*}$.

Proposition 2.1. Let $W$ be a simple $\mathfrak{h}$-module. Suppose that $\mathfrak{n}_{1} \cdot W^{*}$ contains no h-modules isomorphic with $W$. Then $W^{*}$ is simple.

Proof. Let $M$ be a nonzero $(3)$-submodule of $W^{*}$. Let $P$ and $Q$ be as above. By Lemma $2.1(4), P(M) \neq(0)$. Thus $P(M)=W^{* n_{2}}$ since $W^{* n_{2}}$ is simple. If $M \neq W^{*}$ then there is a $v \in M$ such that $P(v) \neq 0$ and $Q(v) \neq 0$. In fact, if there is a $v \in M$ such that $Q(v)=0, P(v) \neq 0$ then $v \in W^{* \mathrm{n}_{2}}$ and by definition of $W^{*}$ and simplicity of $W, U(\mathrm{~B}) \cdot v$ $=W^{*}$. Let $v \in M$ such that $P(v) \neq 0$ and $Q(v) \neq 0$. If $h \cdot P(v) \neq 0$ and $h \cdot Q(v)=0$ for some $h \in U(\mathfrak{h})$ then by the above argument $M=W^{*}$. Thus there is an element $v$ in $M$ such that $P(v) \neq 0$ and such that if $h \cdot P(v) \neq 0$ then $h \cdot Q(v) \neq 0$. Now let $\tilde{W}=U(\mathfrak{h}) \cdot Q(v) \subset \mathfrak{n}_{1} \cdot W^{*}$. We define a map $\xi: \tilde{W} \rightarrow W^{* \pi_{2}}$ by setting $\xi(h \cdot Q(v))=h \cdot P(v)$ for each $h \in U(\mathfrak{h})$. We show that $\xi$ is well defined. If $h \cdot Q(v)=h^{\prime} \cdot Q(v)$ then $\left(h-h^{\prime}\right) Q(v)=0$. Thus $\left(h-h^{\prime}\right) P(v)=0$ and thus $\xi(h \cdot Q(v))=\xi\left(h^{\prime} \cdot Q(v)\right)$. Thus $\xi$ is well defined and injective. $\xi$ is now clearly an $\mathfrak{h}$-module isomorphism. This contradiction implies that $M=W^{*}$. 
3. An imprimitivity theorem. In this section we prove the converse of Lemma 2.1. We maintain the notation of $\$ 2$.

THEOREM 3.1. If $V$ is a (\$-module such that

(1) $V^{\mathfrak{n}_{2}} \neq(0)$ if $V \neq(0)$,

(2) $U\left(\right.$ (\$) $\cdot V^{\mathrm{n}_{2}}=V$,

(3) $\mathfrak{n}_{1} \cdot V \cap V^{\mathfrak{n}_{2}}=(0)$,

(4) $\mathfrak{n}_{1} \cdot V$ contains no nonzero (S)-submodules of $V$ then $V$ is (S)-isomorphic with $\left(V^{\mathbf{n}_{2}}\right)^{*}$.

Proof. By the P-B-W theorem $U(\mathfrak{s})=U\left(\mathfrak{n}_{2}+\mathfrak{h}\right)+\mathfrak{n}_{1} \cdot U\left(\mathfrak{n}_{1}\right) U\left(\mathfrak{n}_{2}+\mathfrak{h}\right)$ a left $\mathfrak{h}$-module direct sum. Let $\gamma_{1}: U(\mathfrak{s}) \rightarrow U\left(\mathfrak{n}_{2}+\mathfrak{h}\right)$ and $\gamma_{2}: U(\mathfrak{S})$ $\rightarrow \mathfrak{n}_{1} \cdot U\left(\mathfrak{n}_{1}\right) U\left(\mathfrak{n}_{2}+\mathfrak{h}\right)$ be the corresponding $\mathfrak{h}$-module projections. Suppose that $v \in V$. Then $v=g \cdot \tilde{v}$ for some $\tilde{v} \in V^{\mathfrak{n}_{2}}$ by (2). Thus $v=\gamma_{1}(g) \cdot \tilde{v}+\gamma_{2}(g) \cdot \tilde{v}$ with $\gamma_{1}(g) \cdot \tilde{v} \in V^{\mathfrak{n}_{2}}$ and $\gamma_{2}(g) \cdot \tilde{v} \in \mathfrak{n}_{1} \cdot V$. Now (3) implies that $V=V^{\mathfrak{n}_{2}} \oplus \mathfrak{n}_{1} \cdot V$ an h-module direct sum. Let $R: V \rightarrow V^{\mathfrak{n}_{2}}$ be the corresponding $\mathfrak{h}$-module projection. We define $\delta: V \rightarrow T\left(V^{\mathfrak{n}_{2}}\right)$ by $\delta(v)(g)=R(g \cdot v)$. Then $\delta(v)(k g)=R(k g \cdot v)=0$ if $k \in \mathfrak{n}_{1} \cdot U(\xi) . \delta(v)(k g)$ $=R(k g \cdot v)=k \cdot R(g v)$ if $k \in \mathfrak{h}$. Thus $\delta(v) \in T(W)$. Now $\delta\left(g_{0} \cdot v\right)(g)$ $=R\left(g g_{0} \cdot v\right)=\delta(v)\left(g g_{0}\right)=\left(g_{0} \cdot \delta(v)\right)(g)$ and thus $\delta: V \rightarrow T(V)$ is a (\$)module homomorphism. If $\delta(v)=0$ then $\delta(v)(g)=0$ for all $g \in U(\xi)$ and thus $R(g \cdot v)=0$ for all $g \in U(\$)$. This says that $U\left((\xi) \cdot v \subset \mathfrak{n}_{1} \cdot V\right.$ and hence $v=0$. Thus $\delta$ is injective. Suppose that $v \in V^{\mathfrak{n}_{2}}$ then $\delta(v)(g)$ $=R(g \cdot v)=\gamma(g) \cdot v$ (here $v$ is looked upon as an element of $\left.V^{\mathfrak{n}_{2}}\right)^{\wedge}$. Thus $\left.\delta\right|_{V} ^{n_{2}}=w$. This clearly implies that

$$
\delta(V)=U\left((\$) w\left(V \mathfrak{n}_{2}\right)=\left(V \mathfrak{n}_{2}\right)^{*} \quad\right. \text { Q.E.D. }
$$

As a corollary to Theorem 3.1 we derive the main result of [5] without using the technique of "double dualization."

Corollary 3.1. Suppose that $V$ is a finite dimensional simple (5)module and that $\mathfrak{n}_{1}$ and $\mathfrak{n}_{2}$ act nilpotently on $V$. Then $V$ is isomorphic with $\left(V^{\mathfrak{m}_{2}}\right)$ * as a (\$-module.

Proof. We show that $V$ satisfies conditions (1)-(4) of Theorem 3.1. If $V=(0)$ then $V=(0)^{*}$. If $V \neq(0)$ then by assumption $V^{\mathfrak{m}_{2}} \neq(0)$. Since $V$ is simple, $U(\mathfrak{b}) \cdot V^{\mathfrak{n}_{2}}=V$. If $\mathfrak{n}_{1} \cdot V \cap V^{\mathfrak{n}_{2}} \neq(0)$ then

$$
V=U(\mathfrak{\$}) \cdot\left(\mathfrak{n}_{1} \cdot V \cap V \mathfrak{n}_{2}\right)=U\left(\mathfrak{n}_{1}\right) \cdot\left(\mathfrak{n}_{1} \cdot V \cap V \mathfrak{n}_{2}\right) \subset U\left(\mathfrak{n}_{1}\right) \mathfrak{n}_{1} \cdot V .
$$

But $\mathfrak{n}_{1}$ acts nilpotently on $V$; thus the above inclusion implies the contradiction $V=(0)$. Thus $\mathfrak{n}_{1} \cdot V \cap V^{\mathfrak{n}_{2}}=(0)$. Finally, since $\mathfrak{n}_{1} \cdot V$ is a proper subspace of $V, \mathfrak{n}_{1} \cdot V$ cannot contain any nonzero (b)-submodule of $V$. 
4. The standard decomposition of a semisimple Lie algebra. In this section we assume that $(5)$ is semisimple and that $K$ is of characteristic 0 and algebraically closed. Let $\mathfrak{h}$ be a Cartan subalgebra of (5) and let $\Delta$ be the root system of (5) with respect to $\mathfrak{h}$. That is, let $\Delta$ be the set of all linear forms $\alpha$ on $\mathfrak{h}$ such that the set $\mathfrak{S}_{\alpha}=\{x \in(\mathfrak{S})[h, x]$ $=\alpha(h) \cdot x$ for all $h \in \mathfrak{h}\}$ is nonzero. Let $\pi$ be a set of linearly independent elements of $\Delta$ such that every element of $\Delta$ can be written as an integral combination of the elements of $\pi$ with the coefficients all having the same sign. Such systems always exist, see e.g. Jacobson [3]. Let $\succ$ be a linear order on $\Delta$ corresponding to $\pi$ (i.e. $\alpha \succ 0$ if $\alpha=\sum n_{\gamma} \gamma$ sum over $\gamma \in \pi$ with $\left.\sum n_{\gamma}>0\right)$. Let $\mathfrak{n}^{+}=\sum_{\alpha>0} \mathfrak{S}_{\alpha}, \mathfrak{n}^{-}$ $=\sum_{\alpha<0} \mathfrak{H}_{\alpha}$. Then $\mathfrak{G}=\mathfrak{n}^{-} \oplus \mathfrak{h} \oplus \mathfrak{n}^{+}$is a decomposition of $\mathfrak{B}$.

Proposition 4.1. If $W$ is a simple finite dimensional (=1 dimensional) h-module then $W^{*}$ is a simple (3-module.

Proof. $W^{*}=U($ (s) $) W^{* \mathfrak{n}^{+}}=U\left(\mathfrak{n}^{-}\right) \cdot W^{* \mathfrak{n}^{+}}=W^{* \mathfrak{n}^{+}} \oplus \mathfrak{n}^{-} \cdot U\left(\mathfrak{n}^{-}\right) \cdot W^{* \mathfrak{n}^{+}}$. Now consider $\mathfrak{n}^{-} \cdot U\left(\mathfrak{n}^{-}\right)$as an $\mathfrak{h}$-module under the action $\operatorname{ad}(h) n$ $=h \cdot n-n \cdot h$ for $n \in \mathfrak{n}^{-} \cdot U\left(\mathfrak{n}^{-}\right)$.

By the P-B-W theorem $\mathfrak{n}^{-} \cdot U\left(\mathfrak{n}^{-}\right)$is completely reducible as an $\mathfrak{h}$-module and $\mathfrak{n}^{-} \cdot U\left(\mathfrak{n}^{-}\right)=\sum V_{\gamma}$ where $V_{\gamma}=\left\{n \in \mathfrak{n}^{-} \cdot U\left(\mathfrak{n}^{-}\right) \mid \operatorname{ad}(h) \cdot n\right.$ $=\gamma(h) \cdot n, h \in \mathfrak{h}\}$ and the above sum is an $\mathfrak{h}$-module direct sum where the $\gamma$ 's are taken to be all nonnegative integral combinations of positive roots. Furthermore $\operatorname{dim}_{K} V_{\gamma}=p(\gamma) \cdot p(\gamma)$ is the number of ways that $\gamma$ can be written as a sum $\gamma=\gamma_{1}+\cdots+\gamma_{r}$ where $\gamma_{i} \in \Delta$, $0 \prec \gamma_{1} \leqq \gamma_{2} \leqq \gamma_{3} \leqq \ldots \leqq \gamma_{r}$. Thus $\mathfrak{n}^{-} \cdot U\left(\mathfrak{n}^{-}\right) \otimes_{K} W^{* \mathfrak{n}^{+}}$as a Lie algebra tensor product module (i.e. $h \cdot(n \otimes v)=\operatorname{ad}(h) \cdot n \otimes v+n \otimes h \cdot v$ ) is isomorphic with $\sum_{\gamma} p(\gamma) K_{\gamma} \otimes W$ where $K_{\gamma}$ is the h-module $K$ with action $h \cdot 1=\gamma(h), p(\gamma)$ is the corresponding multiplicity. $K_{\gamma} \otimes W$ is a simple h-module. Let $\phi: \mathfrak{n}^{-} \cdot U\left(\mathfrak{n}^{-}\right) \otimes W^{* \mathfrak{n}^{+}} \rightarrow \mathfrak{n}^{-} \cdot U\left(\mathfrak{n}^{-}\right) \cdot W^{* \mathfrak{n}^{+}}$by $\phi(n \otimes v)=n \cdot v$ then $\phi$ is an h-module homomorphism. Thus $\mathfrak{n}^{-} \cdot W^{*}$ $=\mathfrak{n}^{-} \cdot U\left(\mathfrak{n}^{-}\right) \cdot W^{* \mathfrak{n}^{+}}$is $\mathfrak{h}$-isomorphic with $\sum_{\gamma} M_{\gamma} K_{\gamma} \otimes W$ where the $M_{\gamma}$ correspond as above to multiplicities. Let $P_{\gamma}: \mathfrak{n}^{-} \cdot W^{*} \rightarrow M_{\gamma} K_{\gamma} \otimes W$ be the corresponding $\mathfrak{h}$-module projection. Suppose that $\tilde{W}$ is an $\mathfrak{h}$-submodule of $\mathfrak{n}^{-} \cdot W$ such that $\tilde{W}$ is $\mathfrak{h}$-isomorphic with $W$. Let $\boldsymbol{\gamma}$ be such that $P_{\gamma}(\tilde{W}) \neq 0$. Since $\tilde{W}$ is simple $\left.P_{\gamma}\right|_{W}$ is an $\mathfrak{h}$-module injection. Thus $W$ is $\mathfrak{h}$-isomorphic with $K_{\gamma} \otimes W$, for some $\gamma \neq 0$, which is impossible. Thus $W^{*}$ is simple by Theorem 3.1 .

If $W$ is the $\mathfrak{h}$-module $K_{\lambda}$ where $\lambda \in \mathfrak{h}^{0}$, the dual space of $\mathfrak{h}$, and $K_{\lambda}$ is the $\mathfrak{h}$-module $K$ with the action $h \cdot 1=\lambda(h)$, then we set $W^{*}=V^{\lambda}$. We notice that $\left(V^{\lambda}\right)^{\mathfrak{n}^{+}}=\left\{v \in V^{\lambda} \mid h \cdot v=\lambda(h) v, h \in \mathfrak{h}\right\}=w\left(K_{\lambda}\right)$ and thus $\operatorname{dim}\left(V^{\lambda}\right)^{\mathfrak{n}^{+}}=1$. Furthermore $V^{\lambda}=U(\mathfrak{S}) \cdot\left(V^{\lambda}\right)^{\mathfrak{n}^{+}}=U\left(\mathfrak{n}^{-}\right) \cdot\left(V^{\lambda}\right)^{\mathfrak{n}^{+}}$. Thus by the techniques of the proof of Proposition 4.1 we find that $V^{\lambda}$ 
$=\sum_{\mu \in \mathfrak{b}^{0}} V_{\boldsymbol{u}}^{\lambda}$ where $V_{\mu}^{\lambda}=\left\{v \in V^{\lambda} \mid h \cdot v=\mu(h) v\right\}$. And $V_{\mu}^{\lambda} \neq 0$ only if $\lambda-\mu$ is a sum of positive roots. Thus $V^{\lambda}$ is the $\mathfrak{n}^{+}$extreme $\mathfrak{h}$-module with highest weight $\lambda$ as in Jacobson [3] or Sophus Lie [4].

5. A multiplication on $U(\mathbb{S})^{0}$. Let \&s be a Lie algebra over a field $K$. Let $U(\$)$ be its universal enveloping algebra and let $U(\$))^{0}$ be

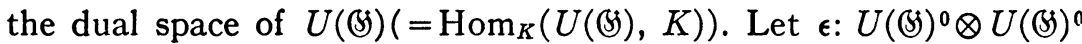
$\rightarrow K$ be given by $\epsilon(f \otimes g)=f(1) g(1)$ and extended by linearity. $U(\mathbb{B})^{0}$ is a left (\$)-module under the action $(x \cdot f)(g)=f(g x)$ where $f \in U(\mathbb{S})^{0}$, $x \in(B), g \in U(\mathbb{B})$. We look upon $U(\mathbb{B})^{0} \otimes U(\mathbb{B})^{0}$ as the tensor product (5)-module (hence $U(\mathbb{B})$-module). We can now define a multiplication on $U(\mathbb{S})^{0}$ : let $f, f^{\prime} \in U(\mathbb{B})^{0}$ then $f \cdot f^{\prime}(g)=\epsilon\left(g \cdot\left(f \otimes f^{\prime}\right)\right)$. It is not hard to check that $U(\mathbb{B})^{0}$ is an associative, commutative ring under this multiplication. $U(\mathbb{S})^{0}$ contains a unit. In fact, $U(\mathbb{S})=K \cdot 1 \oplus(\mathfrak{S} \cdot U(\mathbb{S})$ let $f_{0}: U(\mathbb{S}) \rightarrow K$ be the corresponding projection. Then $f_{0} \cdot f=f$ for all $f \in U(\$))^{0}$. If $K$ is of characteristic 0 then $\left.U(\$)\right)^{0}$ is an integral domain. (If $K$ is of characteristic $p \neq 0$ then $U((B))^{0}$ has no elements $f$ such that $f^{2}=f$.) For details see Cartier [1] or Hochschild [2]. We also note that the map $U(\mathbb{S})^{0} \otimes U(\mathbb{B})^{0} \rightarrow U(\mathbb{B})^{0}$ given by $f \otimes f^{\prime} \rightarrow f \cdot f^{\prime}$ is a $(\$ \text {-module homomorphism. Thus } B \text { acts on } U(\$))^{0}$ as derivations.

We now return to the case (B) semisimple and $K$ characteristic 0 and algebraically closed, and we maintain the notation of $\$ 4$. If $V$ is a ( $)$-module and $\mu \in \mathfrak{h}^{0}$ we set (as before) $V_{\mu}=\{v \in V \mid h \cdot v=\mu(h) \cdot v\}$. We also note that if $\lambda \in \mathfrak{h}^{0}$ then (setting) $T\left(K_{\lambda}\right)=T(\lambda) \subset U(\mathfrak{B})^{0}$. Set

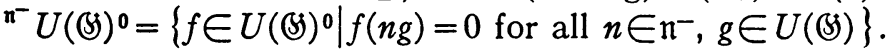

Lemma 5.1. (a) ${ }^{n^{-}} U(\mathbb{S})^{0}$ is a subring of $U(\mathfrak{S})^{0}$.

(b) If $\lambda, \lambda^{\prime}, \mu, \mu^{\prime} \in \mathfrak{h}^{0}$ then $T(\lambda)_{\mu} \cdot T\left(\lambda^{\prime}\right)_{\mu^{\prime}} \subset T\left(\lambda+\lambda^{\prime}\right)_{\mu+\mu^{\prime}}$.

Proof. (a) Let $n \in \mathfrak{n}^{-}, g \in U(\mathfrak{B}), f, f^{\prime} \in \in^{\mathfrak{n}^{-}} U(\mathfrak{H})^{0}$. Then $f \cdot f^{\prime}(n g)$ $=\epsilon\left(n g\left(f \otimes f^{\prime}\right)\right)$. Let $g \cdot\left(f \otimes f^{\prime}\right)=\sum_{i j} f_{i} \otimes f_{j}^{\prime}, f_{j}^{\prime}, f_{i} \in^{n^{-}} U(\mathbb{S})^{0}$ (if $f \in{ }^{\mathfrak{n}^{-}} U(\mathbb{S})^{0}$ then $\left.g \cdot f \in{ }^{\mathfrak{n}^{-}} U(\mathbb{S})^{0}\right) . \quad \epsilon\left(n g\left(f \otimes f^{\prime}\right)\right)=\epsilon\left(n \cdot \sum f_{i} \otimes f_{j}^{\prime}\right)$ $=\epsilon\left(\sum\left(n \cdot f_{i} \otimes f_{j}^{\prime}+f_{i} \otimes n \cdot f_{j}^{\prime}\right)\right)=\sum\left(f_{i}(n) \cdot f_{j}^{\prime}(1)+f_{i}(1) f_{j}^{\prime}(n)\right)=0$. Thus $f \cdot f^{\prime} \in \mathfrak{n}^{-} U(\mathfrak{\$})^{0}$.

(b) is proved similarly.

Let $F(\mathfrak{S})=\left\{f \in^{\mathfrak{n}^{-}} U(\mathbb{S})^{0} \mid \operatorname{dim}_{K} U(\mathbb{S}) \cdot f<\infty\right\}$. Clearly $F(\mathfrak{S})$ is a subalgebra of ${ }^{-} U(\mathfrak{S})^{0}$ and $f_{0} \in F(\mathfrak{B})$.

THEOREM 5.1. $F(\$)$ contains every finite dimensional simple (3module exactly once. Furthermore, $F(\$)=\sum_{\lambda} V^{\lambda}$ a 3 -module direct sum over all $\lambda$ dominant integral (that is if $\alpha \in \pi$ then $2\langle\lambda, \alpha\rangle /\langle\alpha, a\rangle$ is a nonnegative integer). Furthermore $V^{\lambda} \cdot V^{\lambda^{\prime}}=V^{\lambda+\lambda^{\prime}}$ if $\lambda$ and $\lambda^{\prime}$ are dominant integral. 
Proof. Let $V$ be a finite dimensional simple submodule of $F($ (S) . Then $\operatorname{dim}_{K} V^{\mathfrak{n}^{+}}=1$ and there is a dominant integral $\lambda \in \mathfrak{h}^{0}$ such that if $v \in V^{\mathfrak{n}^{+}}$then $h \cdot v=\lambda(h) \cdot v$. If $g \in U(\mathfrak{S}), h \in \mathfrak{h}, f \in V^{\mathfrak{n}^{+}}$then $g=n^{-} h^{\prime} n^{+}$, $n^{-} \in U\left(\mathfrak{n}^{-}\right), h^{\prime} \in U(\mathfrak{h}), n^{+} \in U\left(\mathfrak{n}^{+}\right)$and $f(h g)=f\left(h n^{-} h^{\prime} n^{+}\right)=f\left(n^{-} h h^{\prime} n^{+}\right)$ $=0$ if $n^{-} \neq 1$ and $n^{+} \neq 1$. Thus if $n^{-}$or $n^{+} \neq 1$ then $f(h g)=0=\lambda(h) f(g)$. If $n^{-}=n^{+}=1$ then $f\left(h h^{\prime}\right)=f\left(h^{\prime} h\right)=(h \cdot f)\left(h^{\prime}\right)=\lambda(h) f\left(h^{\prime}\right)$. Thus $f \in T(\lambda)^{\mathfrak{n}^{+}}$ $=\left(V^{\lambda}\right)^{\mathfrak{n}^{+}}$. And thus $V=V^{\lambda}$.

If $f \in F(\$)$ then $\operatorname{dim}_{K} U($ (\$) $) \cdot f<\infty$. Thus $U(\$) \cdot f=\sum V_{i}, V_{i}$ finite

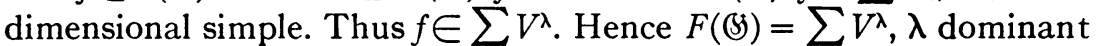
integral.

By Lemma 5.1 we have $V^{\lambda} \cdot V^{\lambda^{\prime}} \subset T\left(\lambda+\lambda^{\prime}\right)$. By the above the only finite dimensional simple submodule of $T\left(\lambda+\lambda^{\prime}\right)$ is $V^{\lambda+\lambda^{\prime}}$. Thus if $\lambda, \lambda^{\prime}$ are dominant integral then $V^{\lambda} \cdot V^{\lambda^{\prime}}=V^{\lambda+\lambda^{\prime}}$.

Let $\pi=\left\{\alpha_{1}, \cdots, \alpha_{l}\right\}$ and let $\lambda_{1}, \cdots, \lambda_{l}$ be defined by $2\left\langle\alpha_{i}, \lambda_{j}\right\rangle /\left\langle\alpha_{i}, \alpha_{i}\right\rangle=\delta_{i j}=0$ if $i \neq j,=1$ if $i=j$. Then by Theorem 5.1 we know that $V^{\lambda_{1}}+V^{\lambda_{2}}+\cdots+V^{\lambda_{l}}$ generates $F(\xi)$. Let $V=V^{\lambda_{1}}+V^{\lambda_{2}}$ $+\cdots+V^{\lambda_{l}}$. Consider the symmetric algebra on $V, S(V), l$-graded in the natural manner. That is $S^{n}(V)$ where $n=\left(n_{1}, \cdots, n_{l}\right), n_{i} \geqq 0$, $n_{i}$ an integer is the space spanned by all products of $n_{1}$ elements of $V^{\lambda_{1}}$, $n_{2}$ elements of $V^{\lambda_{2}}$, etc. $S(V)$ inherits a $(\xi$-module structure from $V$. And there is a natural algebra and (S-homomorphism $\Phi: S(V) \rightarrow F(\$)$ such that $S^{n}(V) \rightarrow V^{n_{1} \lambda_{1}+\ldots+n l \lambda_{l}}$. Since $F(\xi)$ is an integral domain $\operatorname{Ker} \Phi$ is a prime ideal in $S(V)$. If $I(V)=\{f \in S(V) \mid x \cdot f=0$ for all $x \in(S)$ then $I^{+}(V)=I(V) \bigcap_{n \neq 0} S^{n}(V)$ is a subalgebra of $\operatorname{Ker} \Phi$. We have not as yet found the relationship between $\operatorname{Ker} \Phi$ and $I^{+}(V)$.

We conclude this section with a simple example of $\operatorname{Ker} \Phi$. Let (अ) $=A_{2} \cdot \pi=\left\{\alpha_{1}, \alpha_{2}\right\}$. Then $V^{\lambda_{1}}$ is just the 3 dimensional representation of $A_{2}$ as $\operatorname{sl}(3, K)$ and $V^{\lambda_{2}}$ is just the dual module of $V^{\lambda_{1}}$. If $X_{1} \in V_{\lambda_{1}}^{\lambda_{1}}, X_{2} \in V_{\lambda_{1}-\alpha_{1}}^{\lambda_{1}}, X_{3} \in V_{\lambda_{1}-\alpha_{1}-\alpha_{2}}^{\lambda_{1}}$. Identifying $V^{\lambda_{2}}$ with the dual module of $V^{\lambda_{1}}$ we take the dual basis $Y_{1}, Y_{2}, Y_{3}$, of $X_{1}, X_{2}, X_{3} .\left(Y_{1} \in V_{-\lambda_{1}}^{\lambda_{2}}\right.$, $\left.Y_{2} \in V_{\lambda_{2}-\alpha_{2}}^{\lambda_{2}}, Y_{3} \in V_{\lambda_{2}}^{\lambda_{2}}\right)$ and $\operatorname{Ker} \Phi$ is generated by $X_{1} Y_{1}+X_{2} Y_{2}+X_{3} Y_{3}$.

\section{BIBLIOGRAPHY}

1. P. Cartier, Dualité de Tannaka des groupes et des algèbres de Lie, C. R. Acad. Sci. Paris 242 (1956), 322-325.

2. G. Hochschild, Algebraic Lie algebras and representative functions, Illinois J. Math. 3 (1959), 499-523.

3. N. Jacobson, Lie algebras, Interscience, New York, 1962.

4. Séminaire Sophus Lie, Mimeographed notes, Ann. Sci. Ecole Norm. Sup., Paris, 1954-1955.

5. N. Wallach, Induced representations of Lie algebras and a theorem of Borel-Weil, Trans. Amer. Math. Soc. 136 (1969), 181-187.

University of California, Berkeley 